\title{
Cervical Myelopathy Secondary to Atlas Hypoplasia - Reports of 3 Adult Cases -
}

\author{
Han Chang*, Woo-Jin Cho*, Jong-Beom Park ${ }^{\dagger}$, Myung-Sang Moon*, \\ Jong-Woo Chae*, Jong-Seon Ryu*, Hyun-Suk Kang* \\ *Spine Center, Department of Orthopedic Surgery, Sun General Hospital, Daejeon, Korea, \\ †Department of Orthopedic Surgery, Uijongbu St. Mary's Hospital, The Catholic University of Korea, \\ School of Medicine, Seoul, Korea
}

There have been paucity of reports on atlas hypoplasia, and as a result this condition is not clearly defined, nor well understood. The authors reported three cases of atlas hypoplasia that were found in adults who presented with myelopathic symptoms. On radiographic examination, it was found that the anterior-posterior diameter of the atlas was remarkably narrower in all three cases in comparison with normal persons. The MRI in all three cases also revealed intramedullary high signal lesions at the levels where severe spinal cord compression was present. This led to our diagnosis of atlas hypoplasia causing myelopathy.

Key Words: Atlas hypoplasia, Cervical myelopathy, Cervical spinal stenosis

\section{Introduction}

Congenital spinal canal stenosis is one pathomechanism that causes cervical myelopathy. Most cervical spinal stenosis generally occurs below the level of $\mathrm{C}^{1}$, but occasionally is found above. Myelopathy resulting from upper cervical spinal stenosis due to a defective posterior $\operatorname{arch}^{2,3}$, and a hypoplastic atlas ring with a completely developed posterior arch has been reported.

Although a few cases of hypoplastic atlas in children have been reported ${ }^{4}$ to the best of our knowledge, there have only been 10 reported cases worldwide in adults with this condition $^{5-7}$.

\section{Case Reports}

Case 1

A 79 year old male presented complaining of clumsiness in both hands and walking difficulty.

The symptoms developed spontaneously three months prior to the initial visit without any trauma, which aggravated over the last month. Walking was almost impossible without assistance and the clumsiness was most severer in the right hand.

Physical examination revealed that in the right upper extremity all muscle power below the level of the trapezius markedly decreased to 'poor,' while decreasing slightly to 'good' in the left upper extremity. In the right lower extremities muscle power below the right hip decreased to 'poor.' Sensory examination revealed hypesthesia in the region below the level of the $\mathrm{C} 5$ dermatome.

The patient exhibited finger escape sign in the right hand, and the grip and release test showed a decrease to one time within 10 seconds in the right hand and 10 times in the left hand within 10 seconds (normal value: over 20 times in 10 seconds). Simple lateral radiographs showed severe atlas hypoplasia and degenerative changes at all cervical levels. Even though atlantodental interval (ADI) was increased to $3.5 \mathrm{~mm}$, there was no finding of severe instability on flex-

Corresponding author: Han Chang, MD

Spine Center, Department of Orthopedic Surgery, Sun General Hospital

10-7 Mok-dong, Joon-gu, Daejeon, Korea

Tel: +82-42-220-8150, Fax: +82-42-254-4955, E-mail: chspine@korea.com 
ion-extension lateral radiographs (Fig. 1A). The configuration of the upper cervical spine could be clearly seen in the preoperative three-dimensional computed tomography (3D$\mathrm{CT}$ ) images. The atlas ring was quite smaller than normal, and severe compression of the spinal cord by soft tissue posterior to the odontoid process of $\mathrm{C} 2$, as well as an intramedullary high signal intensity area could be observed in T2-MRI. Space available for the cord (SAC) in the atlas was measured to be $6.9 \mathrm{~mm}$. (Fig. 1B and C).

Surgical treatment was performed by doing a posterior $\mathrm{C} 1$ ring laminectomy (Fig. 1D). Complete decompression could be confirmed by postoperative MRI (Fig. 1E). The patient's preoperative JOA score of 3 points recovered up to 11 points postoperatively.

\section{Case 2}

A 49 year old male presented complaining of a squeezing feeling at the midsection and tingling sensations in both upper and lower extremities.

Diagnosis with ossification of the posterior longitudinal ligament (OPLL) of the cervical spine was made before hospital admission. The symptoms started to develop approximately three months prior to the initial visit. There was no difficulty in walking, but the patient showed Lhemitte's sign when extending the neck abruptly. The patient denied any sensory and motor change on physical examination. Simple lateral radiographs revealed OPLL in the region of $\mathrm{C} 2$ through $\mathrm{C} 5$ together with atlas hypoplasia (Fig. 2A). Magnetic resonance imaging showed spinal canal stenosis from $\mathrm{C} 1$ to $\mathrm{C} 5$, and especially marked narrowing of the spinal canal with cord compression at the atlas ring and an intramedullary high signal intensity area (Fig. 2B). Surgical decompressive laminectomy of the posterior $\mathrm{C} 1$ ring was performed, relieving compression of the spinal cord, which was thought to be the main cause of the symptoms. A preoperative JOA score of fifteen points recovered up to sixteen points within a year after the operation, and the postoperative cervical condition was observed well maintained in simple lateral radiographs taken fourteen months after the operation (Fig. 2C). In spite of sufficient decompression of the spinal cord on the postoperative MRI, an intramedullary high signal intensity area was still present at the previous region (Fig. 2D).
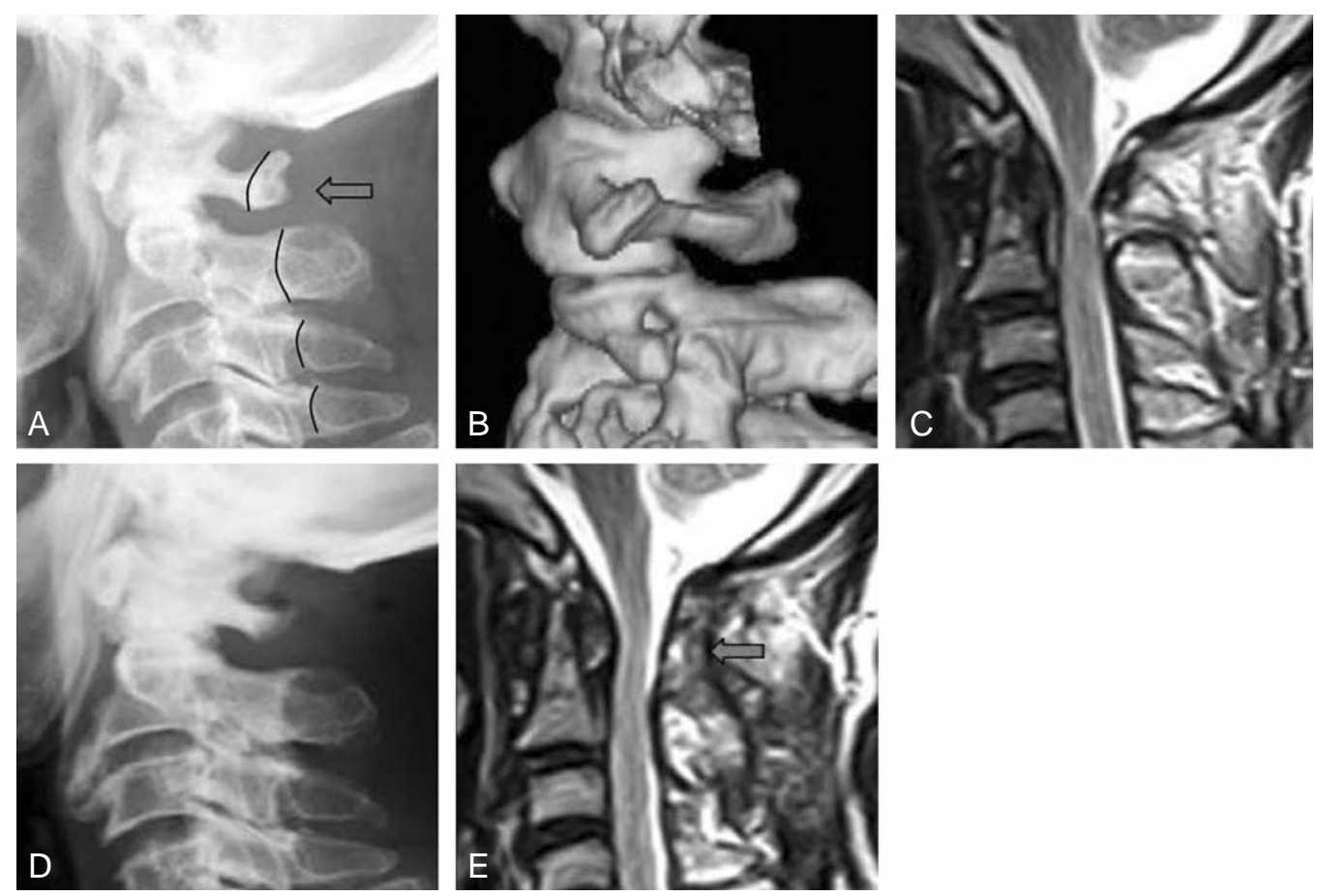

Fig. 1. A seventy-nine year old male with cervical myelopathy. (A) Pre-operative lateral radiograph shows marked narrowing of the atlas ring and disruption of the spinolaminar line $(\boldsymbol{\sim})$. Pre-operative 3D-CT scan (B) clearly defines atlas hypoplasia, and T2-weighted MRI (C) shows the cord compression at the level of the atlas ring. Post-operative posterior C1 ring laminectomy radiograph (D) and T2-weighted MRI (E) shows the well-decompressed spinal cord and restored subarachnoid space at the level of the previous lesion (4). 


\section{Case 3}

A 62 year old male presented complaining of paresthesia in the fingertips of both hands and feet.

The symptoms started to develop after falling down approximately three months before admission. There was no difficulty in walking, but insignificant clumsiness in fine activities like using chop sticks and buttoning a shirt. Physical examination revealed no definite neurological symptoms except hypesthesia of both hands and feet, nor were any upper motor neuron signs or abnormal reflexes shown. Suboccipital headaches were, however, noted. There were findings of atlas hypoplasia and an increase of ADI to $3.1 \mathrm{~mm}$ shown in simple lateral radiographs, and an angular difference of thirteen degrees was measured in flexion-extension lateral radiographs (Fig. 3A and B). MRI revealed severe compression of the spinal cord by stenosis of the hypoplastic atlas and an intramedullary high signal intensity area (Fig. 3C and D). Marked atlas hypoplasia and stenosis could also be observed in the 3D-CT and axial CT scans
(Fig. 3E and F). As surgical treatment, a posterior $\mathrm{C} 1$ ring laminectomy with autogenous iliac bone graft and occipitoatlanto-axial fusion using wire fixation was performed. Halovest external immobilization was applied postoperatively for three months (Fig. 3G). The patient's symptoms recovered completely from the preoperative JOA score of thirteen points to a JOA score of seventeen points.

\section{Discussion}

To understand the congenital malformation of the atlas, it is required to know about the process of its formation and development. Formation of the atlas starts to develop from the three ossification centers originating from the first sclerotome. Both lateral ossification centers begin growing posteriorly within six to seven weeks of embryogenesis to form the posterior arch and unite against each other within four months. The anterior center, however, forms part of the anterior tubercle and arch, and uniting with the two lateral
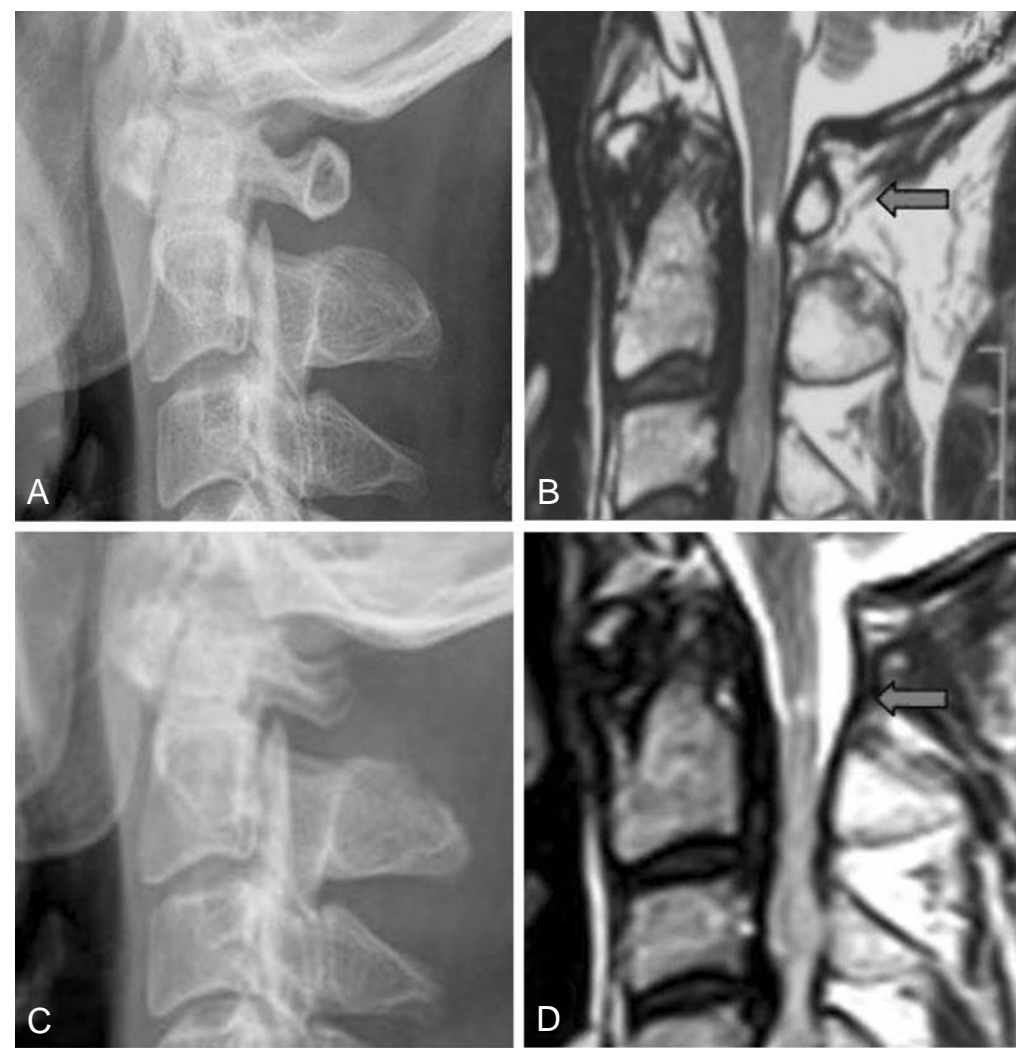

Fig. 2. Forty-nine year old male with cervical myelopathy. (A) Pre-operative lateral radiograph shows hypoplasia of the atlas and ossification of the posterior longitudinal ligament at sub-axial region. (B) T2-weighted MRI shows compression of spinal cord at the level of C1-2 ( $)$ ). (C) Post-operative (posterior C1 ring laminectomy) radiograph. (D) Post-operative T2-weighted MRI shows the well-decompressed spinal cord and restored subarachnoid space, but still present intramedullary high signal at the level of previous lesion $(\boldsymbol{\bullet})$. 
ossification centers at six or seven years after birth ${ }^{8}$. Therefore, it is considered that the cause of atlas hypoplasia may be premature fusion or incomplete posterior development in the process of union of the two halves ${ }^{5}$. It is reported that the average AP diameter of the normal atlas is $16 \sim 20 \mathrm{~mm}$, and it is generally known that clinical symptoms appear below $10 \mathrm{~mm}$ in diameter ${ }^{7}$. The first report about atlas hypoplasia was made by Wackenheim ${ }^{5}$, who reported a case of atlas hypoplasia having occipital neuralgia, but not accompanying myelopathy. The first report of atlas hypoplasia with myelopathy was made by Sawada et al. ${ }^{7}$ who reported one case of surgical treatment for a patient having only $7 \mathrm{mms}$ of anterior-posterior diameter of the spinal canal.

One case report each by Nishikawa et al. ${ }^{5}$ and Tokiyoshi followed and two additional cases by Phan et al. ${ }^{6}$ were reported, and most recently Nichikawa et al. ${ }^{5}$ reported three cases. All these 10 cases are thought to have myelopathy due to atlas hypoplasia. In addition, there are a few case reports of myelopathy caused by atlas hypoplasia associated with pathology of surrounding structures ${ }^{9,10}$. Sato et al. ${ }^{9}$ reported two cases of myelopathy by hypertrophy of the odontoid process of the $\mathrm{C} 2$ spine and hypertrophic osteoarthritis of the C1-2 lateral mass. Yamashita et al. ${ }^{10}$ reported a case of myelopathy by atlas hypoplasia associated with pseudotumor posterior to the odontoid process, which was similar to one of the current case series, but it differed in that the current case had no trauma. All together the current 3 cases add up just a few to the previously reported cases. As can be seen in the three current cases, the authors ' have shown myelopathy by a compressed spinal cord due to atlas hypoplasia. In Case 1, soft tissue swelling posterior to the odontoid process of the $\mathrm{C} 2$ spine was associated, and in Case 2, ossification of the posterior longitudinal ligament at the subaxial region was associated. In Case 3 , in spite of the spinal stenosis throughout the entire cervi-
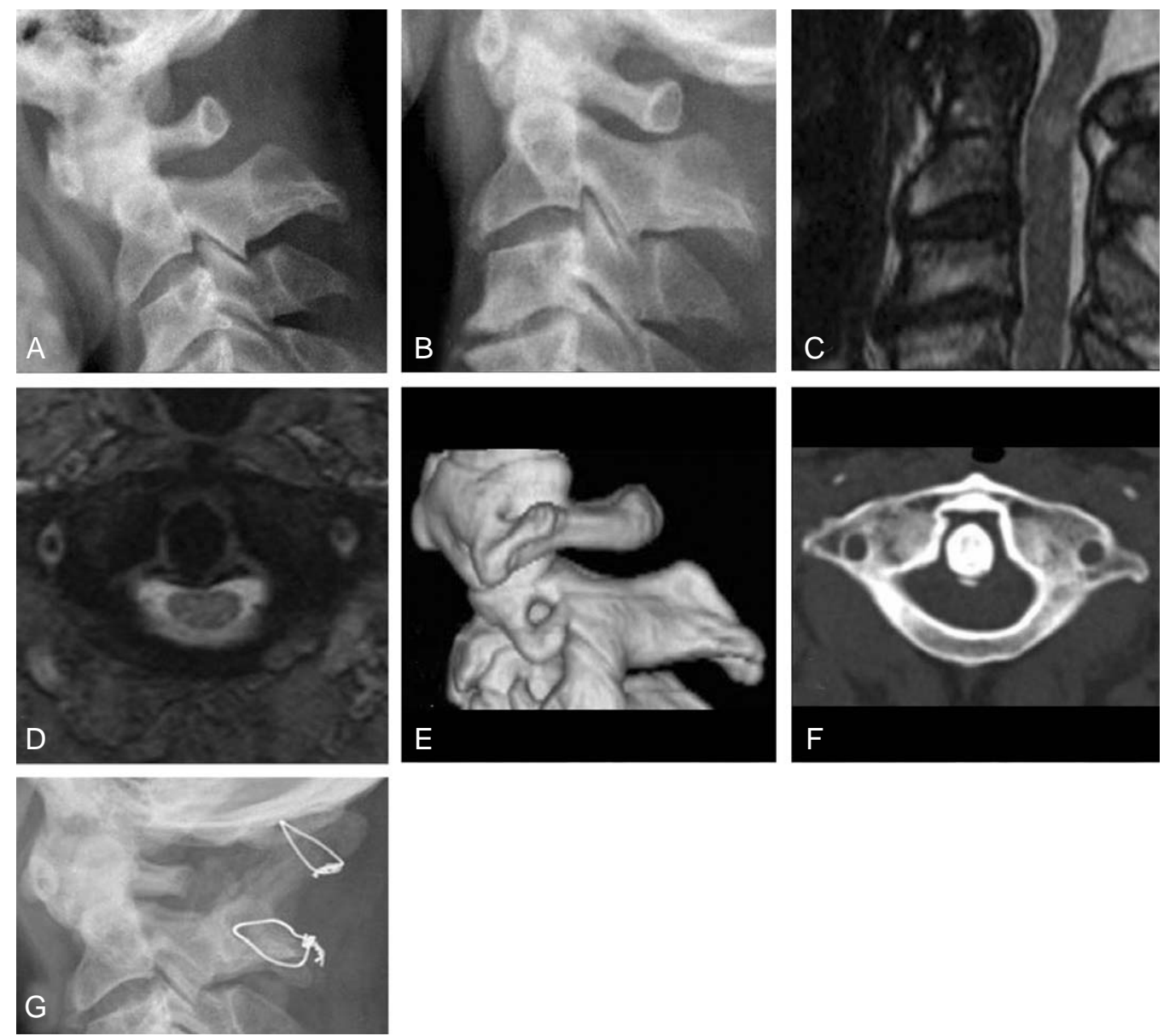

Fig. 3. Sixty-two year old male complaint of paresthesia in the fingertips of both hands and feet. (A, B) Flexion-extension lateral radiographs show marked narrowing of the atlas ring and atlantoaxial instability. (C, D) T2-weighted sagittal and axial MRI show cord compression at the level of atlas. Pre-operative 3D-CT (E) and axial CT (F) scans show small and narrow atlas ring. (G) Postoperative (posterior $\mathrm{C} 1$ ring laminectomy and occipitocervical fusion) one year, lateral radiograph shows a well united C0-2. 
cal spine, the assumed main cause of the myelopathy was the stenosis of the atlas because of the intramedullary high signal lesion located anterior to the posterior ring of the atlas in T2 MRI. In the current 3 cases, intramedullary high signal lesions at the maximum compression level were observed at the compressed part of the spinal cord, and decompression through the posterior $\mathrm{C} 1$ ring laminectomy was performed accordingly in all cases. In Case 3, because of instability in preoperative flexion-extension lateral radiographs, an occipitocervical fusion was performed after a $\mathrm{C} 1$ ring laminectomy and then Halovest external immobilization was applied.

In the current authors' three cases, all of the patients' symptoms were recovered remarkably.

There are very few incidences of myelopathy due to atlas hypoplasia worldwide. By adding these three cases, the authors in treating myelopathic patients suggested to include atlas hypoplasia observed in simple lateral radiographs as one differential diagnosis. Also the authors think that long-term follow up of these and other cases is necessary to better understand the reason why this disease develops in adults only, and not in children, and why it develops spontaneously without any trauma, even though it is assumed to be a congenital malformation. Added with other cases, it may be necessary to establish a disease entity by analyzing common aspects of the natural process of the development of myelopathy in patients with atlas hypoplasia.

\section{REFERENCES}

1. Epstein JA, Carras R, Hyman RA, Costa S: Cervical myelopathy caused by developmental stenosis of the spinal canal. J Neurosurg 1979; 51: 362-367.

2. Currarino G, Rollins N, Diehl JT: Congenital defects of the posterior arch of the atlas: a report of seven cases including an affected mother and son. AJNR Am J Neuroradiol 1994; 15: 249-254.

3. Klimo P Jr, Blumenthal DT, Couldwell WT: Congenital partial aplasia of the posterior arch of the atlas causing myelopathy: case report and review of the literature. Spine 2003; 28: E224-228.

4. Devi BI, Shenoy SN, Panigrahi MK, Chandramouli BA, Das BS, Jayakumar PN: Anomaly of arch of atlas--a rare cause of symptomatic canal stenosis in children. Pediatr Neurosurg 1997; 26: 214-217.

5. Nishikawa K, Ludwig SC, Colon RJ, Fujimoto Y, Heller JG: Cervical myelopathy and congenital stenosis from hypoplasia of the atlas: report of three cases and literature review. Spine 2001; 26: E80-86.

6. Phan N, Marras C, Midha R, Rowed D: Cervical myelopathy caused by hypoplasia of the atlas: two case reports and review of the literature. Neurosurgery 1998; 43 : 629-633.

7. Sawada H, Akiguchi I, Fukuyama H, Kameyama M, Koyama T: Marked canal stenosis at the level of the atlas. Neuroradiology 1989; 31: 346-348.

8. Vangilder JC, Menezes AH: Craniovertebral junction abnormalities. Clin Neurosurg 1983; 30: 514-530.

9. Sato K, Senma S, Abe E, Saito H, Saito H: Myelopathy resulting from the atlantodental hypertrophic osteoarthritis accompanying the dens hypertrophy. Two case reports. Spine 1996; 21: 1467-1471.

10. Yamashita K, Aoki Y, Hiroshima K: Myelopathy due to hypoplasia of the atlas. A case report. Clin Orthop Relat Res 1997; 338: 90-93. 